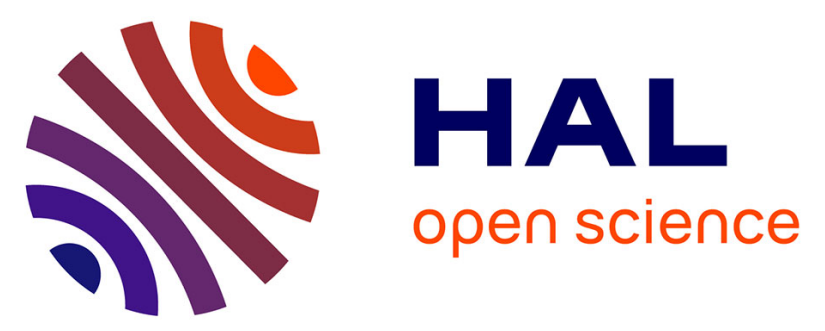

\title{
Universal Conductance Fluctuations in Epitaxial GaMnAs Ferromagnets: Dephasing by Structural and Spin Disorder
}

L. Vila, R. Giraud, L. Thevenard, A. Lemaître, F. Pierre, J. Dufouleur, D. Mailly, B. Barbara, G. Faini

\section{To cite this version:}

L. Vila, R. Giraud, L. Thevenard, A. Lemaître, F. Pierre, et al.. Universal Conductance Fluctuations in Epitaxial GaMnAs Ferromagnets: Dephasing by Structural and Spin Disorder. Physical Review Letters, 2007, 98 (2), pp.027204. 10.1103/PhysRevLett.98.027204 . hal-01659222

\section{HAL Id: hal-01659222 \\ https://hal.science/hal-01659222}

Submitted on 8 Dec 2017

HAL is a multi-disciplinary open access archive for the deposit and dissemination of scientific research documents, whether they are published or not. The documents may come from teaching and research institutions in France or abroad, or from public or private research centers.
L'archive ouverte pluridisciplinaire HAL, est destinée au dépôt et à la diffusion de documents scientifiques de niveau recherche, publiés ou non, émanant des établissements d'enseignement et de recherche français ou étrangers, des laboratoires publics ou privés. 


\title{
Universal Conductance Fluctuations in Epitaxial GaMnAs Ferromagnets: Dephasing by Structural and Spin Disorder
}

\author{
L. Vila, ${ }^{1}$ R. Giraud, ${ }^{1}$ L. Thevenard, ${ }^{1}$ A. Lemaitre, ${ }^{1}$ F. Pierre, ${ }^{1}$ J. Dufouleur, ${ }^{1}$ D. Mailly, ${ }^{1}$ B. Barbara, ${ }^{2}$ and G. Faini ${ }^{1}$ \\ ${ }^{1}$ Laboratoire de Photonique et de Nanostructures-CNRS, Route de Nozay, 91460 Marcoussis, France \\ ${ }^{2}$ Laboratoire de Magnétisme Louis Néel-CNRS, BP166, 38042 Grenoble Cedex-09, France
}

(Received 12 July 2006; published 12 January 2007)

\begin{abstract}
Mesoscopic transport measurements reveal a large effective phase coherence length in epitaxial GaMnAs ferromagnets, contrary to usual $3 d$-metal ferromagnets. Universal conductance fluctuations of single nanowires are compared for epilayers with a tailored anisotropy. At large magnetic fields, quantum interferences are due to structural disorder only, and an unusual behavior related to hole-induced ferromagnetism is evidenced, for both quantum interferences and decoherence. At small magnetic fields, phase coherence is shown to persist down to zero field, even in presence of magnons, and an additional spin disorder contribution to quantum interferences is observed under domain walls nucleation.
\end{abstract}

DOI: 10.1103/PhysRevLett.98.027204

PACS numbers: 75.45.+j, 71.70.- d, 75.50.Lk

Quantum corrections to the conductance were deeply investigated in nonmagnetic metals for two decades [1,2]. It is now well known that quantum interferences of free carriers in a weakly disordered metallic nanostructure affect transport properties of a mesoscopic conductor, e.g., giving rise to universal conductance fluctuations (UCF) under an applied magnetic field or to the weak localization of carriers at low fields [3,4]. In particular, these quantum corrections were used to address decoherence phenomena in a Fermi sea at subkelvin temperatures, which are dominated by Coulomb interactions in pure nonmagnetic metals. Yet, the role of spin was mainly limited to two specific cases: first, the weak localization correction becomes an antilocalization for a strong spinorbit coupling; second, efficient spin-flip scattering can strongly alter phase coherence [5,6], leading to an apparent saturation of the decoherence time at very low temperatures (see [7] and references therein). To avoid such paramagnetic fluctuations, which scale down the inelastic mean-free path (and, therefore, the phase coherence length), one can investigate the opposite regime when ferromagnetic exchange interactions are large enough to freeze single-spin fluctuations. However, quantum transport in a ferromagnet may still be hampered by very efficient decoherence mechanisms, mainly due to lowenergy collective spin fluctuations (magnons) and to fluctuations of inhomogeneous magnetization states (domain walls).

Recently, a few theoretical [8-13] and experimental [14-18] reports studied mesoscopic transport properties of itinerant ferromagnets. Although theory predicted a specific behavior, due to the interplay between exchange and spin-orbit interactions, for either weak localization [10], Aharonov-Bohm oscillations [11,12], or anisotropic UCF [13], the crucial issue of how to preserve phase coherence in a ferromagnet was often put aside. Yet, only very short phase coherence lengths $L_{\phi}$ were reported from experiments for different $3 d$-metal ferromagnets, $L_{\phi}$ being usually smaller than $30 \mathrm{~nm}$ at $T=30 \mathrm{mK}$ [14-18]. In these granular materials, only a small quantum correction to the conductance was observed, together with a much larger classical contribution due to anisotropic magneto conductance. Indeed, weak decoherence in a ferromagnet should require both the freezing of spin waves excitations and a saturated magnetization at grain boundaries, though their relative role on decoherence is not known. In the opposite regime of paramagnetism, a short phase coherence length was also found in a diluted magnetic semiconductor (DMS) [19] due to a strong decoherence induced by single-spin fluctuations.

In this work, we demonstrate that both the crystalline quality and the anisotropy act on quantum interferences in a ferromagnet. From UCF measurements, we evidence the unusually large phase coherence length in an epitaxial ferromagnetic DMS, GaMnAs [20], with $L_{\phi} \approx 100 \mathrm{~nm}$ at $T \approx 100 \mathrm{mK}$, which is preserved down to zero field. Based on a comparison between epilayers having either an in-plane or a perpendicular magnetic anisotropy, the contributions from both structural and spin disorder to UCF can be separated. At large applied fields, quantum interferences are only due to structural disorder and show a behavior specific to hole-induced ferromagnetism in a DMS, quite similarly to a recent study of ultranarrow GaMnAs nanowires [21]. Besides, we found that the decoherence mechanism has no apparent dimensional crossover and seems to be independent of the anisotropy. At small fields and for an in-plane anisotropy, the nucleation of rare domain walls clearly affects the correlation field of UCF, although their amplitude is not modified. This strongly suggests that such a weak spin disorder contributes to dephasing without increasing decoherence significantly, even in presence of magnons.

High quality GaMnAs epilayers, $50 \mathrm{~nm}$ thick and with a Mn content of about 6\%, were grown at low temperature 
( $T \approx 250^{\circ} \mathrm{C}$ ) over either an undoped GaAs buffer layer (compressive strains, in-plane anisotropy) or an undoped InGaAs one (tensile strains, perpendicular anisotropy), previously elaborated at high temperature (see Ref. [22] for details). Highly $p$-doped samples were obtained after annealing at $T \approx 250^{\circ} \mathrm{C}$ for an hour, the Curie temperature thus rising from about $70 \mathrm{~K}$ to $130 \mathrm{~K}$. A hole density $p \approx 5 \times 10^{20} \mathrm{~cm}^{-3}$ was deduced from high-field Hall measurements, together with a typical resistivity $\rho \approx$ $2 \mathrm{~m} \Omega \mathrm{cm}$ at $T=100 \mathrm{mK}$ for annealed samples $\left(R_{\square} \approx\right.$ $500 \Omega$ ). Despite a much lower carrier density, the mobility is quite comparable to a metal, and a rough estimation of the diffusion constant gives $D \approx 10^{-4} \mathrm{~m}^{2} \mathrm{~s}^{-1}$. Narrow nanowires were patterned by $e$-beam lithography and Arplasma etching, with a length and a width down to $200 \mathrm{~nm}$ and $50 \mathrm{~nm}$, respectively. The smallest dimension remains longer than the screening length to avoid complications due to edge depletion (of about 5-10 nm). Ohmic contacts were made by $\mathrm{Ti} / \mathrm{Au}$ deposition. Low-noise four-probe transport measurements were performed down to the base temperature $T_{\text {cryo }} \approx 25 \mathrm{mK}$ of a dilution refrigerator, using a lock-in technique and a compensating loop to improve signal to noise ratio, stability, and resolution. A magnetic induction up to $7 \mathrm{~T}$ was applied perpendicularly to the sample plane. All measurements were done with a lowenough voltage bias $V$ to avoid any increase in the electronic temperature $\left(e V \leq k_{B} T\right)$ [23].

For a highly degenerate GaMnAs nanowire, the temperature dependence of the resistivity shows a metalliclike behavior below the Curie temperature (cusp at $T_{C} \approx$ $130 \mathrm{~K}$, see Fig. 1, top), with an increase below about $10 \mathrm{~K}$ probably due to Coulomb interactions [23]. The only difference between GaMnAs epilayers having either an in-plane or a perpendicular anisotropy is related to the evolution of the magnetization under a perpendicular applied field. This results in a different classical contribution to the magnetoconductance below $1 \mathrm{~T}$, as measured with a large applied bias $\left(e V / k_{B} T \approx 100\right.$ for $I=100 \mathrm{nA}$, at $T=$ $100 \mathrm{mK}$ ). For an in-plane anisotropy, a positive anisotropic magnetoresistance (AMR) is observed when the magnetization is continuously rotated out of the plane up to the anisotropy induction $B_{A} \approx 0.5 \mathrm{~T}$ (see Fig. 1, bottom left). For a perpendicular anisotropy, the low-field AMR response does not exist anymore, since the magnetization direction is always perpendicular to the current flow. The strong extraordinary Hall voltage reveals an abrupt magnetization jump at the coercive induction of about $25 \mathrm{mT}$ (see Fig. 1, bottom right). The latter is controlled by the nucleation field of a domain wall, which is typical of a magnetization reversal with a small magnetic viscosity (low density of pinning centers). The strong uniaxial anisotropy also opens a gap of about $3 \mathrm{~K}$ in the magnon dispersion curve, so that all spin excitations are frozen at $25 \mathrm{mK}$. In both cases, the high-field negative AMR contribution to the resistance becomes smaller than quantum corrections in the mesoscopic regime, contrary to the case
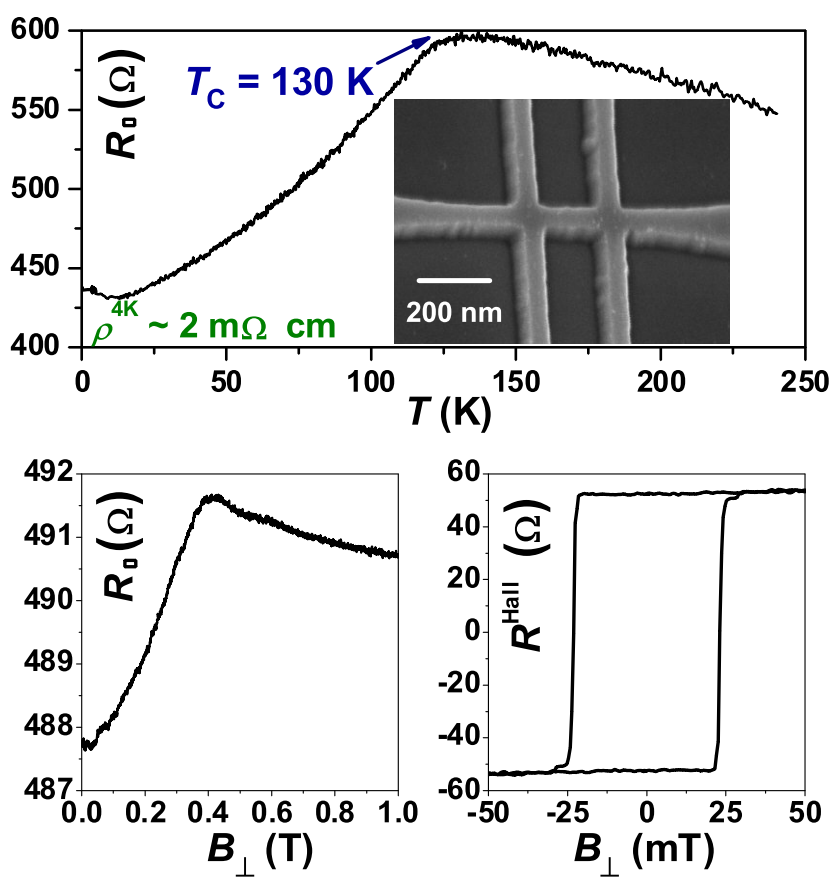

FIG. 1 (color online). (Top) Temperature dependence of the square resistance measured with a GaMnAs nano-Hall bar $(L=$ $220 \mathrm{~nm}, W=50 \mathrm{~nm}$ ), a common behavior for both anisotropy. (Bottom left) Anisotropic magnetoresistance at $T_{\text {cryo }}=25 \mathrm{mK}$, for an in-plane anisotropy. (Bottom right) Extraordinary Hall resistance at $T_{\text {cryo }}=25 \mathrm{mK}$, for a perpendicular anisotropy. All measurements were done with a large current $I=100 \mathrm{nA}$, leading to vanishingly small quantum corrections (see text).

of $3 d$ metals, as a consequence of a much larger phase coherence length.

We first focus on GaMnAs nanowires with a perpendicular anisotropy, so that the magnetization remains fully saturated down to the remnant state. At $T_{\text {cryo }} \approx 25 \mathrm{mK}$, reproducible conductance fluctuations are observed when sweeping the magnetic induction between 0 and $7 \mathrm{~T}$, back and forth. Figure 2 shows such four scans, each one being recorded in about 12 hours. The measurement can be reproduced at will (two lower curves), until the temperature is risen (two upper curves). After a thermal cycling up to $4.2 \mathrm{~K}$ (top thick line), a small evolution of microscopic disorder slightly modifies the UCF fingerprint. A much stronger effect is observed after a thermal cycling up to $300 \mathrm{~K}$ (top thin line). As expected in disordered conductors, a new frozen configuration of structural disorder results in a complete change of the magnetofingerprint, the UCF mean amplitude and correlation field remaining unchanged.

Conductance fluctuations were further investigated at higher temperatures (see Fig. 3, top) or larger bias, both resulting in a reduction of the amplitude, as expected for UCF. In the regime $L \geq L_{\phi}$, Fig. 3, bottom shows the scaling behavior of the root mean square deviation to the average conductance, independent of the nanowire length $L$ after renormalization by $L^{3 / 2}$. No saturation was ob- 


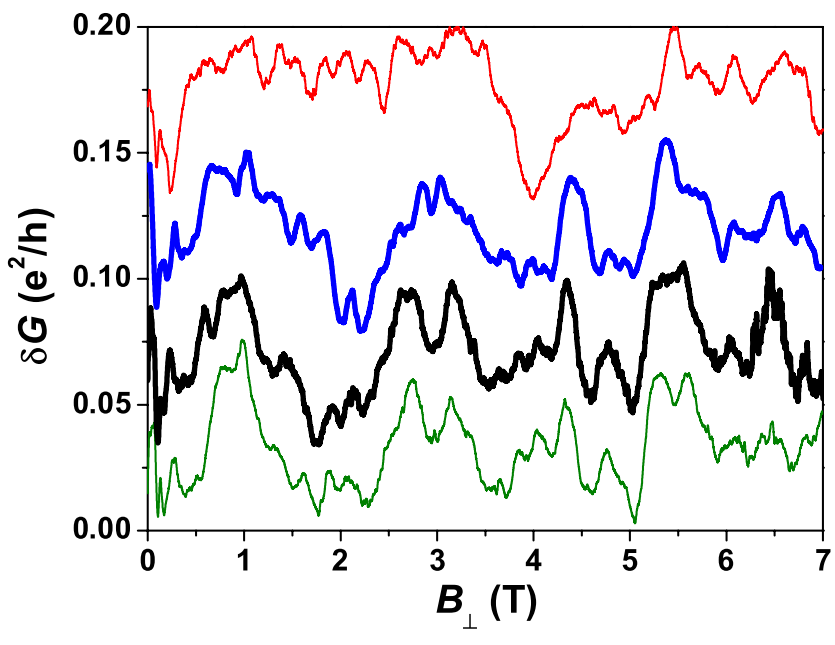

FIG. 2 (color online). (Bottom thick line) UCF of a nanowire with a perpendicular anisotropy $(L=1200 \mathrm{~nm}, W=150 \mathrm{~nm})$ measured at base temperature $T_{\text {cryo }} \approx 25 \mathrm{mK}$, with a small current $I=1 \mathrm{nA}$ corresponding to an electronic temperature $T_{\mathrm{el}} \approx 100 \mathrm{mK}$ [23]. (Bottom thin line) Same measurement, $24 \mathrm{~h}$ later. (Top thick line) Same measurement, after a thermal cycling up to $T=4.2 \mathrm{~K}$. (Top thin line) Same measurement, after a thermal cycling up to $T=300 \mathrm{~K}$.

served down to our lowest electronic temperature $T_{\mathrm{el}} \approx$ $100 \mathrm{mK}$. Surprisingly, the power-law temperature dependence of the scaling function, with $\delta G^{\mathrm{rms}} L^{3 / 2} \propto 1 / T^{\alpha}$ and $\alpha=0.75 \pm 0.15$, does not exhibit a dimensional crossover as a function of temperature or of the wire width, for both widths $W=50 \mathrm{~nm}$ and $W=150 \mathrm{~nm}$. As the expected thermal length $L_{T}$ is about $200 \mathrm{~nm}$ at $T=100 \mathrm{mK}$ and $60 \mathrm{~nm}$ at $T=1 \mathrm{~K}$, such a crossover from a quasi-1D regime $\left(L_{T} \geq W\right)$ to a quasi-2D one $\left(L_{T} \leq W\right)$ should occur for the larger width $W=150 \mathrm{~nm}$, contrary to our observations.

Recently, a very similar result on decoherence was obtained by another group [21] on ultranarrow nanowires $(W=20 \mathrm{~nm})$ having a planar anisotropy, with an identical power-law exponent but with a smaller amplitude of UCF. This can be expected from edge roughness in narrow nanowires, especially when their width is comparable to the screening length, as in Ref. [21]. In our work, the amplitude yields a three times larger phase coherence length, with $L_{\phi} \approx 100 \mathrm{~nm}$ at $T \approx 100 \mathrm{mK}$, for both in-plane and perpendicular anisotropy. Such a large value is corroborated by measurements on shorter wires, showing a progressively larger amplitude of UCF, which already reaches the quantum of conductance for a nanowire length $L=$ $220 \mathrm{~nm}$ (see Fig. 3, top), that is, when $L$ becomes comparable to $L_{\phi}$. Note that only some effective values of $L_{\phi}$ are extracted from the amplitude of UCF using semiclassical results for a quasi-1D behavior [3,4], with $\min \left(L_{T}, L_{\phi}\right) \geq$ $W$. Similar values are obtained in two opposite regimes, using either $\delta G^{\mathrm{rms}}=\left(e^{2} / h\right)\left(L_{\phi} / L\right)^{3 / 2}$ (for $L_{T} \geq L_{\phi}$ ) or $\delta G^{\mathrm{rms}}=\left(e^{2} / h\right)\left(L_{T} / L\right)\left(L_{\phi} / L\right)^{1 / 2}$ (for $\left.L_{T} \leq L_{\phi}\right)$. This is not a surprise since $L_{T} \approx L_{\phi}$ for GaMnAs, which further
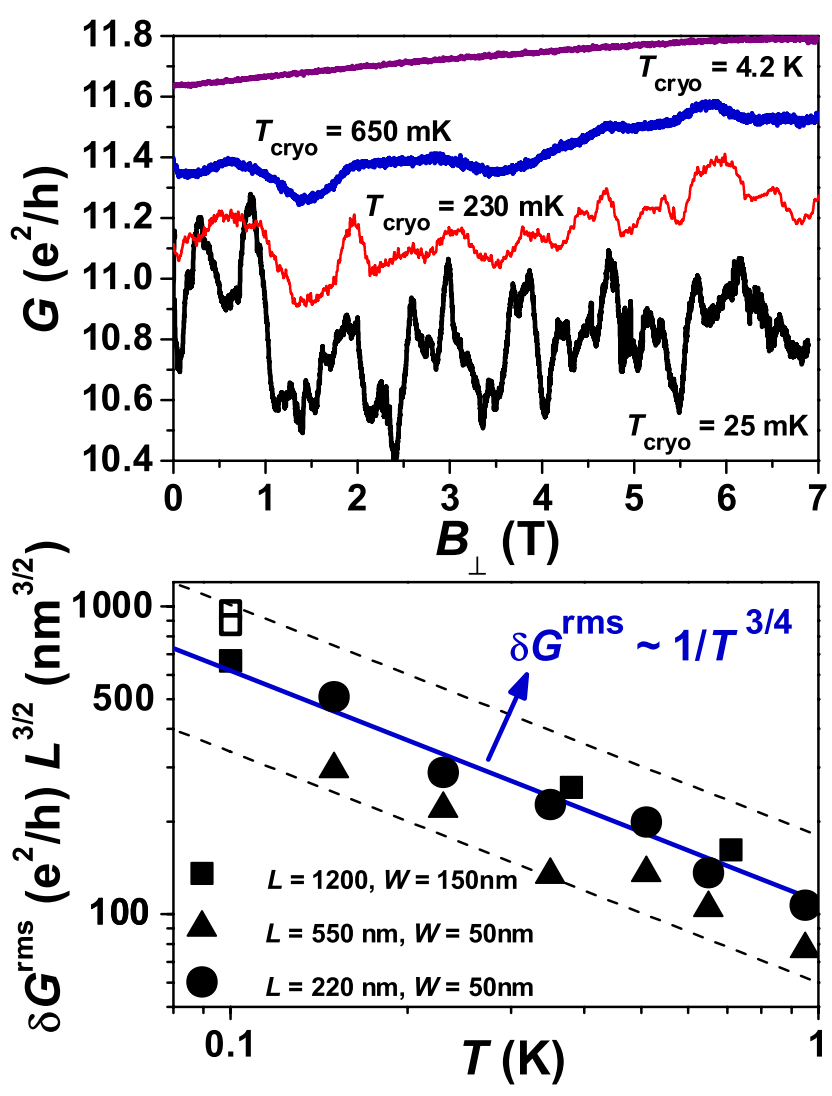

FIG. 3 (color online). (Top) Temperature dependence of UCF for a short nanowire with a perpendicular anisotropy $(L=$ $220 \mathrm{~nm}, W=50 \mathrm{~nm}$ ). (Bottom) Temperature dependence of the normalized root mean square UCF amplitude $\delta G^{\mathrm{rms}} L^{3 / 2}$, showing a universal scaling law $1 / T^{3 / 4}$. Dashed lines define the accuracy for the determination of the prefactor. All results correspond to a perpendicular anisotropy, but open squares (planar anisotropy, $L=1200 \mathrm{~nm}$ and $600 \mathrm{~nm}, W=150 \mathrm{~nm}$ ).

complicates the semiclassical analysis. Both results give $L_{\phi} \propto 1 / \sqrt{T}$. Note that the diffusion constant is an important limitation to even longer coherence lengths in ferromagnetic DMS.

Deeper insights on the nature of UCF in GaMnAs nanowires were obtained from a detailed comparison between epilayers with either an in-plane or a perpendicular anisotropy (see Fig. 4). Whereas UCF remain the same down to zero field for the magnet with a strong perpendicular anisotropy (constant and uniform magnetization), a clear crossover is observed below the anisotropy field for an inplane anisotropy. Indeed, when the magnetization rotates back to the sample plane, much faster conductance fluctuations are observed (see Fig. 4 inset). This results from the influence of magnetic domain walls (spin disorder) on UCF. Just below the anisotropy field, a few domain walls are very likely to spontaneously nucleate on rare strong pinning centers, giving a reproducible spin contribution to UCF (see the inset in Fig. 4, with two successive sweeps, starting from $7 \mathrm{~T}$ ). Remarkably, the amplitude of conductance fluctuations remains the same at low fields, which strongly suggests that the phase coherence length is neither 


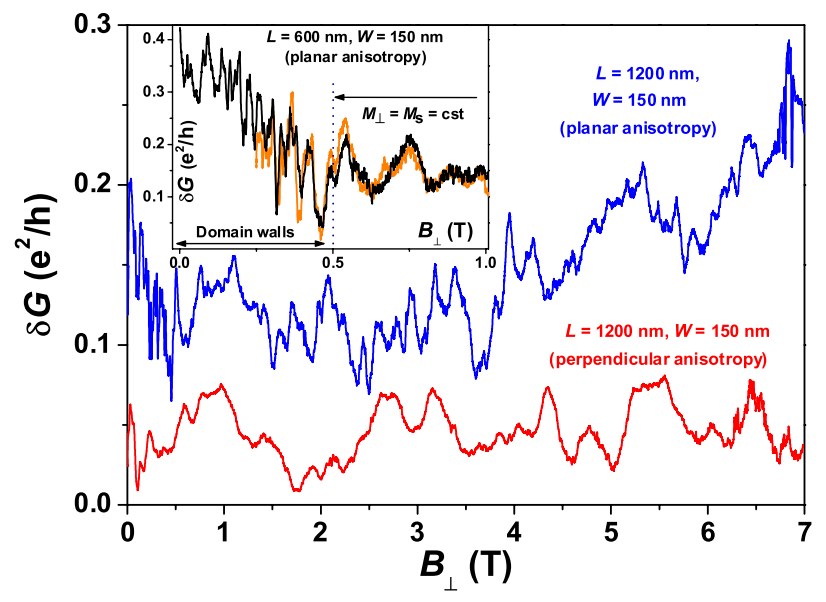

FIG. 4 (color online). UCF measured at base temperature for a nanowire with either a perpendicular or a planar anisotropy. (Inset) Crossover below the anisotropy field for a planar anisotropy, showing the effect of domain walls on quantum interferences. Each field sweep begins from $7 \mathrm{~T}$.

affected by the presence of a couple of domain walls nor by magnons. Domain walls also lead to a hysteretic behavior, so that unreproducible fluctuations are observed when measuring minor loops.

The comparison between different anisotropies further reveals some striking deviations from the standard UCF behavior known in nonmagnetic metals, even when only structural disorder remains at large fields (perpendicular magnetization and strongly reduced spin-flip scattering, in any case). For instance, there is not a one-to-one relationship between the amplitude of UCF and a given correlation field. Although not understood in detail at present, such a specific behavior in GaMnAs epilayers can be expected for hole-induced ferromagnetism: (i) up to four valence subbands can contribute to hole transport, possibly giving different correlation fields, and their relative position is modified by epitaxial strains; (ii) the semiclassical approximation may fail since $k_{F} l$ is only a few units, because of a rather long Fermi wavelength, even in the strongly $p$-doped regime. This makes the exact determination of the phase coherence length even more tedious, and only some effective values are derived from a semiclassical analysis of the UCF amplitude.

To sum up, we evidenced a large phase coherence length in epitaxial GaMnAs ferromagnets, making a clear distinction between the structural and spin disorder contributions to UCF. Effective values of $L_{\phi}$ were extracted from the amplitude of UCF, using semiclassical results. However, the analysis of the correlation fields is nontrivial, due to the contribution of four valence subbands. Also, the nature of an unusual decoherence mechanism remains unclear, with no apparent dimensional crossover for nanowires with a width as large as $150 \mathrm{~nm}$. From a comparison between similar epilayers only differing by their anisotropy, we give the first direct evidence of free carriers dephasing by magnetic domain walls. Importantly, no significant decoherence from domain walls or magnons was found in GaMnAs nanowires.

O. Mauguin and L. Largeau are acknowledged for X-ray characterizations of our (Ga, Mn)As epilayers, A. Miard for epitaxial growth, and L. Leroy and L. Couraud for their technical assistance. Financial support by the Conseil Général de l'Essonne and by the national project on nanoscience No. NR216 "PARCOUR" are gratefully acknowledged.

[1] Y. Imry, Introduction to Mesoscopic Physics (Oxford University Press, Oxford, 1997).

[2] E. Akkermans and G. Montambaux, Mesoscopic Physics of Electrons and Photons (EDP Sciences, Paris, 2006).

[3] B. L. Altshuler and A. G. Aronov, in Electron-Electron Interaction in Disordered Conductors, edited by A.L. Efros and M. Pollak (Elsevier Science Publishers B.V., Amsterdam, 1985).

[4] P. A. Lee, A. D. Stone, and H. Fukuyama, Phys. Rev. B 35, 1039 (1987).

[5] A. Benoit, S. Washburn, R. A. Webb, D. Mailly, and L. Dumoulin, in Anderson Localization, edited by T. Ando and H. Fukuyama (Springer, New York, 1988).

[6] A. Benoit, D. Mailly, P. Perrier, and P. Nédellec, Superlattices Microstruct. 11, 313 (1992).

[7] F. Pierre et al., Phys. Rev. B 68, 085413 (2003).

[8] G. Tatara and H. Fukuyama, Phys. Rev. Lett. 78, 3773 (1997).

[9] Y. Lyanda-Geller, I. L. Aleiner, and P. M. Goldbart, Phys. Rev. Lett. 81, 3215 (1998).

[10] V. K. Dugaev, P. Bruno, and J. Barnás, Phys. Rev. B 64, 144423 (2001).

[11] G. Tatara and B. Barbara, Phys. Rev. B 64, 172408 (2001).

[12] G. Tatara, H. Kohno, E. Bonet, and B. Barbara, Phys. Rev. B 69, 054420 (2004).

[13] S. Adam, M. Kindermann, S. Rahav, and P. W. Brouwer, Phys. Rev. B 73, 212408 (2006).

[14] K. Hong and N. Giordano, Phys. Rev. B 51, 9855 (1995).

[15] M. Aprili, J. Lesueur, L. Mumoulin, and P. Nédellec, Solid State Commun. 102, 41 (1997).

[16] S. Kasai, E. Saitoh, and H. Miyajima, J. Appl. Phys. 93, 8427 (2003).

[17] S. Lee, A. Trionfi, and D. Natelson, Phys. Rev. B 70, 212407 (2004).

[18] Y. G. Wei, X. Y. Liu, L. Y. Zhang, and D. Davidovic, Phys. Rev. Lett. 96, 146803 (2006).

[19] J. Jaroszyński et al., Phys. Rev. Lett. 75, 3170 (1995).

[20] H. Ohno, J. Magn. Magn. Mater. 200, 110 (1999).

[21] K. Wagner et al., Phys. Rev. Lett. 97, 056803 (2006).

[22] L. Thevenard et al., Phys. Rev. B 73, 195331 (2006).

[23] A minimum electronic temperature $T_{\mathrm{el}} \approx 100 \mathrm{mK}$ at the cryostat base temperature was inferred from the saturation of the longitudinal resistance $R_{\text {long }}$ measured on long nanowires to average out the UCF contribution. Besides, $R_{\text {long }}$ has an inverse square-root temperature dependence, as expected from Coulomb interactions. 